INJE-TP-02-01, hep-th/0201176

\title{
Absorption cross section in de Sitter space
}

\author{
Y.S. Myung* \\ Relativity Research Center and School of Computer Aided Science, Inje University, Gimhae \\ 621-749, Korea
}

\begin{abstract}
We study the wave equation for a minimally coupled massive scalar in threedimensional de Sitter space. We compute the absorption cross section to investigate its cosmological horizon in the southern diamond. Although the absorption cross section is not defined exactly, we can be determined it from the fact that the low-energy $s(j=0)$-wave absorption cross section for a massless scalar is given by the area of the cosmological horizon. On the other hand, the low-temperature limit of $j \neq 0$-mode absorption cross section is useful for extracting information surrounding the cosmological horizon. Finally we mention a computation of the absorption cross section on the CFT-side using the dS/CFT correspondence.
\end{abstract}

Compiled at October 23, 2018: 629.

*Email-address : ysmyung@physics.inje.ac.kr 


\section{INTRODUCTION}

Recently an accelerating universe has proposed to be a way to interpret the astronomical data of supernova [1-3]. The inflation is employed to solve the cosmological flatness and horizon puzzles arisen in the standard cosmology. Combining this observation with the need of inflation leads to that our universe approaches de Sitter geometries in both the infinite past and the infinite future [4-6]. Hence it is very important to study the nature of de Sitter (dS) space and the dS/CFT correspondence $[7,8]$. However, there exist difficulties in studying de Sitter space. First there is no spatial infinity and global timelike Killing vector. Thus it is not easy to define conserved quantities including mass, charge and angular momentum appeared in asymptotically de Sitter space. Second the dS solution is absent from string theories and thus we do not have a definite example to test the dS/CFT correspondence. Finally it is hard to define the $S$-matrix because of the presence of the cosmological horizon.

We remind the reader that the cosmological horizon is very similar to the event horizon in the sense that one can define its thermodynamic quantities using the same way as was done for the black hole. Two important quantities in the black hole physics are the BekensteinHawking entropy and the absorption cross section (=greybody factor). The former relates to the intrinsic property of the black hole itself, while the latter relates to the effect of spacetime curvature. Explicitly the greybody factor for the black hole arises as a consequence of scattering off the gravitational potential surrounding the horizon [9]. For example, the low-energy $s$-wave greybody factor for a massless scalar has a universality such that it is equal to the area of the horizon for all spherically symmetric black holes [10]. In this work we assume that this universality is valid for the cosmological horizon. The entropy for the cosmological horizon was discussed in literature [11]. However, as far as we know, there is no any explicit computation of the greybody factor for the cosmological horizon ${ }^{1}$.

In this paper we compute the absorption cross section of a massive scalar in the background of three-dimensional de Sitter space. For this purpose we first analyze the wave equation only in the southern diamond where the time evolution of waves is properly defined. We wish to calculate the outgoing flux near $r=0$. And then we compute the outgoing flux by using the matching region of overlapping validity near the cosmological horizon of $r_{c}=1$. Here we follow the conventional approach for a computation of the greybody factor of the black hole.

The organization of this paper is as follows. In section II we briefly review the wave equation in de Sitter space. We perform a potential analysis to study the asymptotic region by introducing a tortoise coordinate $r^{*}$ in section III. In section IV we calculate the flux at $r=0,1$ to obtain the greybody factor. Finally we discuss our results in section VI.

\footnotetext{
${ }^{1}$ Recently a similar work for four-dimensional Schwarzschild-de Sitter black hole appeared in [12]. But it considered mainly the black hole temperature. Also the absorption rate for the Kerr-de Sitter black hole was discussed in [13].
} 


\section{WAVE EQUATION IN DE SITTER SPACE}

We start with the wave equation for a massive scalar

$$
\left(\nabla^{2}-m^{2}\right) \Phi=0
$$

in the background of three-dimensional de Sitter space expressed in the static coordinates

$$
d s_{d S_{3}}^{2}=-\left(1-\frac{r^{2}}{\ell^{2}}\right) d t^{2}+\left(1-\frac{r^{2}}{\ell^{2}}\right)^{-1} d r^{2}+r^{2} d \phi^{2} .
$$

Here $\ell$ is the curvature radius of de Sitter space and hereafter we set $\ell=1$ for simplicity unless otherwise stated. The above metric is singular at the cosmological horizon, which divides space into four regions. There are two regions with $0 \leq r \leq 1$ which correspond to the causal diamonds of observers at the north and south poles : northern diamond (ND) and southern diamond (SD). Two regions with $1<r<\infty$ containing the future-null infinity $\mathcal{I}^{+}$and past-null infinity $\mathcal{I}^{-}$are called future triangle $(\mathrm{FT})$ and past triangle $(\mathrm{PT})$. On $\mathcal{I}^{ \pm}$ the metric (2) is conformal to the cylinder. A timelike Killing vector $\frac{\partial}{\partial t}$ is future-directed only in the southern diamond. To obtain the greybody factor, we have to get a definite wave propagation as time evolves. Hence in this work we confine ourselves to the southern diamond. This means that our working space is compact, in contrast to the case of the black hole. This will give rise to an ambiguity to derive an exact form of the greybody factor.

In connection with the dS/CFT correspondence, one may classify the mass-squared $m^{2}$ into three cases [8]: $m^{2} \geq 1, \quad 0<m^{2}<1, m^{2}=0$. For a massive scalar with $m^{2} \geq 1$, one has a non-unitary $\mathrm{CFT}^{2}$. A scalar with mass $0<m^{2}<1$ can be related to a unitary CFT. A massless scalar with $m^{2}=0$ is special and it would be treated separately. For our purpose we consider $m^{2}$ as a parameter at the beginning. Assuming a mode solution

$$
\Phi(r, t, \phi)=f(r) e^{-i \omega t} e^{i j \phi}
$$

Eq.(1) leads to the differential equation for $r$ [15]

$$
\left(1-r^{2}\right) f^{\prime \prime}(r)+\left(\frac{1}{r}-3 r\right) f^{\prime}(r)+\left(\frac{\omega^{2}}{1-r^{2}}-\frac{j^{2}}{r^{2}}-m^{2}\right) f(r)=0
$$

where the prime $\left(^{\prime}\right)$ denotes the differentiation with respect to its argument.

\section{POTENTIAL ANALYSIS}

We observe from Eq.(4) that it is not easy to understand how scalar waves propagate in the southern diamond. In order to do that, we must transform the wave equation into the Schrödinger-like equation using a tortoise coordinate $r^{*}$ [14]. Then we can get wave forms in

\footnotetext{
${ }^{2}$ Although this belongs to one of examples of non-unitary theories that are dual to well-behaved stable bulk theory, but the connection between the bulk theory and its non-unitary boundary theory is not understood clearly up to now.
} 
asymptotic regions of $r^{*} \rightarrow \pm \infty$ through a potential analysis. We introduce $r^{*}=g(r)$ with $g^{\prime}(r)=1 / r\left(1-r^{2}\right)$ to transform Eq.(4) into the Schrödinger-like equation with the energy $E=\omega^{2}$

$$
-\frac{d^{2}}{d r^{* 2}} f+V(r) f=E f
$$

with a potential

$$
V(r)=\omega^{2}+r^{2}\left(1-r^{2}\right)\left[m^{2}+\frac{j^{2}}{r^{2}}-\frac{\omega^{2}}{1-r^{2}}\right]
$$

Considering $r^{*}=g(r)=\int g^{\prime}(r) d r$, one finds

$$
r^{*}=\ln r-\frac{1}{2} \ln \left(1-r^{2}\right), \quad e^{2 r^{*}}=\frac{r^{2}}{1-r^{2}}, \quad r^{2}=\frac{e^{2 r^{*}}}{1+e^{2 r^{*}}} .
$$

Here we confirm that $r^{*}$ is a tortoise coordinate such that $r^{*} \rightarrow-\infty(r \rightarrow 0)$, whereas $r^{*} \rightarrow \infty(r \rightarrow 1)$. Let us express the potential as a function of $r^{*}$

$$
V\left(r^{*}\right)=\omega^{2}+\frac{e^{2 r^{*}}}{\left(1+e^{2 r^{*}}\right)^{2}}\left[m^{2}+\frac{1+e^{2 r^{*}}}{e^{2 r^{*}}} j^{2}-\left(1+e^{2 r^{*}}\right) \omega^{2}\right] .
$$

For $m^{2}=1, j=0, \omega=0.1$, the shape of this takes a potential barrier $(\frown)$ located at $r^{*}=0$. On the other hand, for all non-zero $j$, one finds the potential step $(\neg)$ with its height $\omega^{2}+j^{2}$ on the left-hand side of $r^{*}=0$. All potentials decrease exponentially to zero as $r^{*}$ increases on the right-hand side. From the quantum mechanics we always have a well-defined wave near the cosmological horizon of $r^{*}=\infty$. But near the coordinate origin of $r^{*}=-\infty(r=0)$ it is not easy to develop a genuine traveling wave.

Near $r=0\left(r^{*}=-\infty\right)$ one finds the equation

$$
\frac{d^{2}}{d r^{* 2}} f_{(-\infty)}-j^{2} f_{(-\infty)}=0
$$

This gives us a solution

$$
f_{(-\infty)}\left(r^{*}\right)=A e^{j r^{*}}+B e^{-j r^{*}}
$$

which is equivalently rewritten by making use of Eq.(7) as

$$
f_{r=0}(r)=A r^{j}+\frac{B}{r^{j}} .
$$

The first term corresponds to a normalizable mode at $r=0\left(r^{*}=-\infty\right)$, while the second is a non-normalizable, singular mode. As one discards the second term in Eq.(11) for calculating the Bogoliubov transformation [15], the first term is needed for our purpose. Hence we set $B=0$. Also we observe that angular momentum modes play the important role in the computation of the flux.

On the other hand, near the cosmological horizon $r_{c}=1\left(r^{*}=\infty\right)$ one obtains a differential equation 


$$
\frac{d^{2}}{d r^{* 2}} f_{\infty}+\omega^{2} f_{\infty}=0
$$

This has a solution

$$
f_{\infty}\left(r^{*}\right)=C e^{-i \omega r^{*}}+D e^{i \omega r^{*}}
$$

which is equivalently rewritten as

$$
f_{r=1}(r)=C\left(1-r^{2}\right)^{\frac{i \omega}{2}}+D\left(1-r^{2}\right)^{-\frac{i \omega}{2}} .
$$

The first wave (second wave) in Eq.(13) together with $e^{-i \omega t}$ implies the ingoing (outgoing) waves across the cosmological horizon. In order to obtain a connection between $A$ and $C, D$, let us consider the case of $j^{2}>m^{2}$ with $V \approx V_{0}=\omega^{2}+j^{2}$ for $-\infty<r^{*} \leq 0$ and $V \approx 0$ for $0 \leq r^{*}<\infty$ with the energy $E=\omega^{2}<V_{0}$. Also it corresponds to the problem for a potential step $V_{0}$ with $0<E<V_{0}$ in the quantum mechanics. Requiring the conservation of flux at $r^{*}=0$ leads to the asymptotic relations : $C \approx(j-i \omega) A /(-2 i \omega), D \approx(j+i \omega) A /(2 i \omega)$. Although the flux on the left hand side of $r^{*}=0$ is zero due to the real function of Eq.(10), the flux of the right hand side is not zero because of the traveling wave nature of Eq.(13).

Up to now we obtain asymptotic forms of a scalar wave which propagates in the southern diamond of de Sitter space. In order to calculate the absorption cross section, we need to know an explicit form of wave propagation in $0 \leq r \leq 1$. This can be achieved only when solving the differential equation (4) explicitly.

\section{FLUX CALCULATION}

In order to solve equation (4), we first transform it into a hypergeometric equation using $z=r^{2}$. Here the working space still remains unchanged as $0 \leq z \leq 1$ covering the southern diamond. This equation takes a form

$$
z(1-z) f^{\prime \prime}(z)+(1-2 z) f^{\prime}(z)+\frac{1}{4}\left(\frac{\omega^{2}}{1-z}-\frac{j^{2}}{z}-m^{2}\right) f(z)=0 .
$$

Here one finds two poles at $z=0,1(r=0,1)$ and so makes a further transformation to cancel these by choosing

$$
f(z)=z^{\alpha}(1-z)^{\beta} w(z), \quad \alpha_{ \pm}= \pm \frac{j}{2}, \quad \beta_{ \pm}= \pm i \frac{\omega}{2} .
$$

First we choose the case of $\alpha_{+}$and $\beta_{+}$. Then we obtain a hypergeometric equation

$$
z(1-z) w^{\prime \prime}(z)+[c-(a+b+1) z] w^{\prime}(z)-a b w(z)=0
$$

where $a, b$ and $c$ are given by

$$
a=\frac{1}{2}\left(j+i \omega+h_{+}\right), \quad b=\frac{1}{2}\left(j+i \omega+h_{-}\right), \quad c=1+j
$$

with 


$$
h_{+}=1+\sqrt{1-m^{2}}\left(=1+i \sqrt{m^{2}-1}\right), \quad h_{-}=1-\sqrt{1-m^{2}}\left(=1-i \sqrt{m^{2}-1}\right) .
$$

Considering $j=$ integer, one regular solution near $z=0$ to Eq.(15) is given by [16]

$$
f_{+}(z)=A z^{j / 2}(1-z)^{i \omega / 2} F(a, b, c ; z)
$$

with an unknown constant $A$. Also there is the other solution with a logarithmic singularity at $z=0$ as $\tilde{f}_{+}(z)=\tilde{A} z^{j / 2}(1-z)^{i \omega / 2}[F(a, b, c ; z) \ln z+\cdots]$. However, both solutions have vanishing flux at $z=0$, because the relevant part $\left(z^{j / 2}\right)$ is not complex but real.

Concerning the case of $\alpha_{-}$and $\beta_{+}(c=1-j)$, one has one regular solution

$$
f_{-}(z)=E z^{j / 2}(1-z)^{i \omega / 2} F(a+j, b+j, 1+j ; z)
$$

and the other singular solution is given by $\tilde{f}_{-}(z)=\tilde{E} z^{j / 2}(1-z)^{i \omega / 2}[F(a+j, b+j, 1+j ; z) \ln z+$ ...]. These have essentially the same behaviour at $z=0$ as in the case of $\alpha_{+}(c=1+j)$.

Now we are in a position to calculate an outgoing flux at $z=0$ which is defined as

$$
\mathcal{F}(z=0)=\left.2 \frac{2 \pi}{i}\left[f^{*} z \partial_{z} f-f z \partial_{z} f^{*}\right]\right|_{z=0} .
$$

For any kind of real functions near $z=0$ including $f_{ \pm}$and $\tilde{f}_{ \pm}$, the outgoing/ingoing flux is given by

$$
\mathcal{F}_{\text {out } / \text { in }}(z=0)=0 \text {. }
$$

Hence we choose a regular solution of Eq.(20) for further calculation. To obtain a flux at the horizon of $z=1(r=1)$, we first have to use a formula : $F(a, b, c ; z)=\frac{\Gamma(c) \Gamma(c-a-b)}{\Gamma(c-a) \Gamma(c-b)} F(a, b, a+$ $b-c+1 ; 1-z)+\frac{\Gamma(c) \Gamma(a+b-c)}{\Gamma(a) \Gamma(b)}(1-z)^{c-a-b} F(c-a, c-b,-a-b+c+1 ; 1-z)$. Using $1-z \sim e^{-2 r^{*}}$ near $z=1$, one finds from Eq.(20) the following form:

$$
f_{0 \rightarrow 1} \equiv f_{\text {in }}+f_{\text {out }}=H_{\omega, j} e^{-i \omega r^{*}}+H_{-\omega, j} e^{i \omega r^{*}}
$$

where

$$
H_{-\omega, j}=A \alpha_{-\omega, j}, \quad \alpha_{-\omega, j}=\frac{\Gamma(1+j) \Gamma(i \omega) 2^{i \omega}}{\left.\left.\Gamma\left[\left(j+i \omega+h_{+}\right) / 2\right)\right] \Gamma\left[\left(j+i \omega+h_{-}\right) / 2\right)\right]} .
$$

Then we match Eq.(13) with Eq.(24) to yield $C=H_{\omega, j}$ and $D=H_{-\omega, j}$ near the cosmological horizon. Finally we calculate its outgoing flux at $z=1\left(r^{*}=\infty\right)$ as

$$
\mathcal{F}_{\text {out }}(z=1)=\left.\frac{2 \pi}{i}\left[f_{\text {out }}^{*} \partial_{r^{*}} f_{\text {out }}-f_{\text {out }} \partial_{r^{*}} f_{\text {out }}^{*}\right]\right|_{r^{*}=\infty}=4 \pi \omega A^{2}\left|\alpha_{-\omega, j}\right|^{2} .
$$

If one uses Eq.(21) instead of Eq.(20), its outgoing wave near $z=1$ is given by $B \alpha_{-\omega, j} e^{i \omega r^{*}}$. This is the same flux as in Eq.(26) except of replacing $A$ by $B$. 


\section{ABSORPTION CROSS SECTION}

An absorption coefficient by the cosmological horizon is defined formally by

$$
\mathcal{A}=\frac{\mathcal{F}_{\text {out }}(z=1)}{\mathcal{F}_{\text {out }}(z=0)} .
$$

However, we have zero-normalization of $\mathcal{F}_{\text {out }}(z=0)=0$ for de Sitter wave propagation. In this case one cannot define the absorption cross section. Instead we propose an unknown normalization $\mathcal{F}_{\text {out }}(z=0)=F$, where $F$ will be determined by referring the absorption cross section for the low-energy $s(j=0)$-wave. Up to now we do not insert the curvature radius $\ell$ of $\mathrm{dS}_{3}$ space. The correct absorption coefficient can be recovered when replacing $\omega(m)$ with $\omega \ell(m \ell)$. Then the absorption cross section in three dimensions is defined by

$$
\sigma_{a b s}=\frac{\mathcal{A}}{\omega}=\left[\frac{4 \pi \ell A^{2}}{F}\right]\left|\alpha_{-\omega \ell, j}\right|^{2}
$$

where

$$
\left|\alpha_{-\omega \ell, j}\right|^{2}=\frac{|\Gamma(1+j)|^{2}|\Gamma(i \omega \ell)|^{2}}{\left.\mid \Gamma\left[\left(j+i \omega \ell+h_{+}\right) / 2\right)\right]\left.\right|^{2}\left|\Gamma\left[\left(j+i \omega \ell+h_{-}\right) / 2\right]\right|^{2}} .
$$

We calculate the low-energy $s(j=0)$-wave cross section for a massless scalar to determine $F[10]$. This is given by

$$
\sigma_{a b s, j=0}^{m \ell=0, \omega \ell \ll 1}=\pi \ell \frac{A^{2}}{F} \rightarrow \mathcal{A}_{c h}
$$

with the area of the cosmological horizon $\mathcal{A}_{c h}=2 \pi \ell$. Hence the unknown flux $\mathcal{F}(z=0)$ is determined as $F=A^{2} / 2$. We wish to classify the absorption cross section according to values of $m \ell$.

\section{A. $m \ell=0$ case}

This case corresponds to the absorption cross section of a massless scalar. Using $h_{+}=$ $2, h_{-}=0$, one finds

$$
\begin{aligned}
\sigma_{a b s}^{m \ell=0}= & {[8 \pi \ell] \frac{(j !)^{2} \pi}{\omega \ell \sinh [\pi \omega \ell]} } \\
& \times \frac{1}{|\Gamma(1+j / 2+i \omega \ell / 2)|^{2}|\Gamma(j / 2+i \omega \ell / 2)|^{2}} .
\end{aligned}
$$

For $j=1$, this takes the form

$$
\begin{aligned}
\sigma_{a b s, j=1}^{m \ell=0}= & \frac{16 \mathcal{A}_{c h}}{\pi \omega \ell \sinh [\pi \omega \ell]} \\
& \times \frac{(\cosh [\pi \omega \ell / 2])^{2}}{\left(1+(\omega \ell)^{2}\right)} .
\end{aligned}
$$


In order to get a definite expression for the absorption cross section, let us consider the low-energy scattering with small $E=(\omega \ell)^{2}<<1$ (for example, $\omega \ell=0.1$ ). Introducing the temperature of the cosmological horizon $T_{c h}=\frac{1}{2 \pi \ell}$, this limit implies $\omega<<2 T_{c h}$. On the other hand there exist the low-temperature limit of $\omega \ell>>1\left(\omega>>2 T_{c h}\right)^{3}$. In the low-energy limit of $\omega \ell<<1, j=1$-mode reduces to

$$
\sigma_{a b s, j=1}^{m \ell=0, \omega \ell<<1}=\frac{16}{\pi^{2}} \frac{\mathcal{A}_{c h}}{(\omega \ell)^{2}} .
$$

On the other hand, its low-temperature limit is given by

$$
\sigma_{a b s, j=1}^{m \ell=0, \omega \ell>>1}=\frac{8}{\pi} \frac{\mathcal{A}_{c h}}{(\omega \ell)^{3}}
$$

For $j=2$, this leads to

$$
\begin{aligned}
\sigma_{a b s, j=2}^{m \ell=0}= & \frac{64 \mathcal{A}_{c h} \pi}{\omega \ell \sinh [\pi \omega \ell]} \\
& \times \frac{(\sinh [\pi \omega \ell / 2])^{2}}{\left(4+(\omega \ell)^{2}\right)(\pi \omega \ell / 2)^{2}} .
\end{aligned}
$$

In the low-energy limit of $\omega \ell<<1$, this reduces to

$$
\sigma_{a b s, j=2}^{m \ell=0, \omega \ell<<1}=\frac{16 \mathcal{A}_{c h}}{(\omega \ell)^{2}} .
$$

But its low-temperature limit is

$$
\sigma_{a b s, j=2}^{m \ell=0, \omega \ell>>1}=\frac{128}{\pi} \frac{\mathcal{A}_{c h}}{(\omega \ell)^{5}}
$$

B. $0<m \ell<1$

In this case there is no further expression for the absorption cross section because of the real values of $h_{ \pm}=1 \pm \sqrt{1-(m \ell)^{2}}$.

\footnotetext{
${ }^{3}$ In the study of the cosmological horizon, this high frequency approximation is very useful for obtaining the absorption cross section [17]. Considering $\omega=2 \pi \nu \sim 1 / \lambda$, this approximation implies $\lambda<<\ell$, which means that the size of the cosmological horizon is very bigger than the wavelength of a test scalar. Hence the scattering of a scalar off the cosmological horizon is well-defined in the low-temperature limit (that is, high frequency approximation).
} 


$$
\text { C. } m \ell=1 \text { case }
$$

Here we have the absorption cross section

$$
\begin{aligned}
\sigma_{a b s}^{m \ell=1}= & {[8 \pi \ell] \frac{(j !)^{2} \pi}{\omega \ell \sinh [\pi \omega \ell]} } \\
& \times \frac{1}{\left(|\Gamma[(j+1+i \omega \ell) / 2]|^{2}\right)^{2}} .
\end{aligned}
$$

In the low-energy limit of $\omega \ell<<1$, the cross section for $j=1$ reduces to

$$
\sigma_{a b s, j=1}^{m \ell=1, \omega \ell<<1}=\frac{4 \mathcal{A}_{c h}}{(\omega \ell)^{2}}
$$

while its low-temperature limit is

$$
\sigma_{a b s, j=1}^{m \ell=1, \omega \ell>>1}=\frac{2}{\pi} \frac{\mathcal{A}_{c h}}{(\omega \ell)^{3}} .
$$

The low-energy cross section for $j=2$ is given by

$$
\sigma_{a b s, j=2}^{m \ell=1, \omega \ell<<1}=\frac{256}{\pi^{2}} \frac{\mathcal{A}_{c h}}{(\omega \ell)^{2}} .
$$

But the low-temperature cross section leads to

$$
\sigma_{a b s, j=2}^{m \ell=1, \omega \ell>>1}=\frac{128}{\pi} \frac{\mathcal{A}_{c h}}{(\omega \ell)^{5}} .
$$

D. $m \ell>1$

In this case we have to use another expression of $h_{ \pm}=1 \pm i \sqrt{(m \ell)^{2}-1}$. For even $j$, one finds the absorption cross section

$$
\begin{aligned}
\sigma_{a b s, j=e v e n}^{m \ell>1}= & {[8 \pi \ell] \frac{(j !)^{2} \cosh \left[\pi y_{1} / 2\right] \cosh \left[\pi y_{2} / 2\right]}{\pi \omega \ell \sinh [\pi \omega \ell]} } \\
& \times \frac{1}{\left|(j-1) / 2+i y_{1} / 2\right|^{2} \cdots\left|1 / 2+i y_{1} / 2\right|^{2}\left|(j-1) / 2+i y_{2} / 2\right|^{2} \cdots\left|1 / 2+i y_{2} / 2\right|^{2}}
\end{aligned}
$$

with $y_{1}=\omega \ell+\sqrt{(m \ell)^{2}-1}$ and $y_{2}=\omega \ell-\sqrt{(m \ell)^{2}-1}$. For odd $j$, one has

$$
\begin{aligned}
\sigma_{a b s, j=o d d}^{m \ell>1}= & {[8 \pi \ell] \frac{(j !)^{2} \sinh \left[\pi y_{1} / 2\right] \sinh \left[\pi y_{2} / 2\right]}{\pi \omega \ell\left(y_{1} / 2\right)\left(y_{2} / 2\right) \sinh [\pi \omega \ell]} } \\
& \times \frac{1}{\left|(j-1) / 2+i y_{1} / 2\right|^{2} \cdots\left|1+i y_{1} / 2\right|^{2}\left|(j-1) / 2+i y_{2} / 2\right|^{2} \cdots\left|1+i y_{2} / 2\right|^{2}} .
\end{aligned}
$$




\section{DISCUSSION}

We calculate the absorption cross section of a minimally coupled scalar which propagates in the southern diamond of three-dimensional de Sitter space. We expect that higherdimensional cases have similar behaviors because their wave equation takes the nearly same form as in the three-dimensional case in Eq.(4).

One of the striking results is that the low-energy $s(j=0)$-wave absorption of a massless scalar is not defined exactly within our approach. This mainly depends on being unable to calculate its non-zero flux at $r=0(z=0)$. However, using a massless scalar propagation in the symmetric black holes whose cross sections are the area of the event horizon of black hole [10], we calculate the low-energy $s(j=0)$-wave absorption Eq.(30) of a massless scalar in the background of de Sitter horizon.

On the other hand, $j \neq 0$-modes of a scalar wave play the important role in extracting information about the cosmological horizon compared to the black holes. We expect from the black hole analysis that the low-energy limit $(\omega R \rightarrow 0)$ of the $l \neq 0$-angular mode absorption cross section is proportional roughly to $(\omega R)^{4 l} \mathcal{A}_{7 B H}$ for the 7D black holes which was derived from D3-branes [18]. For 5D black holes, it is proportional to $\left(\omega r_{o}\right)^{2 l} \mathcal{A}_{5 B H}[19]$. However, one finds from Eqs.(33), (36), (39) and (41) that those for $j \neq 0$ in the low-energy limit of $\omega \ell<<1$ are given by $1 /(\omega \ell)^{2}$ which implies that the absorption cross section is greater than the area of the cosmological horizon. This is not the promising case that we want to get. From the potential analysis it conjectures that for $j \neq 0$, the low-energy absorption cross section with $E=(\omega \ell)^{2}<<1$ is negligible because a potential step with its height $V_{0}=j^{2}+(\omega \ell)^{2}>>E$ is present on the left hand side. Thus it seems that taking a low-energy limit of the de Sitter absorption cross section for $j \neq 0$-mode is meaningless because it is always greater than $\mathcal{A}_{c h}$. In order to obtain a finite absorption cross section, $\omega \ell$ should be large such as $\omega \ell>>1$. Actually this corresponds to the low-temperature limit of $\omega>>2 T_{c h}$. The low-temperature limit (that is, high frequency approximation) is meaningful in the study of de Sitter space since its cross section appears less than $\mathcal{A}_{c h}$. We find from Eqs.(34), (37), (40) and (42) that the absorption cross section decreases as $j$ increases. This is consistent with the potential analysis which states that for given energy $E=\omega^{2}$, the potential increases as $j$ increases. As a result, the low-temperature limit of $j \neq 0$-mode absorption cross section will be used to extract information about the cosmological horizon.

At this stage we wish to comment on the other approach of Suzuki and Takasugi in deriving the absorption cross section in de Sitter space [17]. Actually a definition of absorption probability Eq.(16) in ref. [17] can be applicable for the non-zero spin field. Hence their approach may be problematic to a spin-zero scalar. However, an interesting fact that the absorption cross section of the cosmological horizon can be well-defined in the low-temperature limit is checked by two approaches independently.

Also we wish to mention the difference between the Bogoliubov coefficient and the greybody factor. In calculating the Bogoliubov coefficient, we have to define two different vacuums by introducing global [20] or Kruskal coordinates [15]. In calculating the Bogoliubov coefficients, one needs only a normalizable mode in Eq.(20). Instead the entire space (SD,ND,FT,PT) normalizable solutions are used for finding thermal state of the cosmological horizon by matching them across the the cosmological horizon. Then we find the thermal nature of the cosmological horizon clearly, for example, Planck (black body) spectrum with 
temperature. In this case the fine effect of scattering is usually neglected [21].

However, de Sitter space gives us a potential surrounding the cosmological horizon and it gives rise to a scattering for a test scalar wave which propagates from $r=0$ to $r=\ell$. We need each non-zero flux at $r=0,1$ in the southern diamond to calculate the transmission (absorption) coefficient. But we have zero-flux at $r=0$. Since the working space is compact and it includes the origin of coordinate $(r=0)$, it may be problematic to derive an exact form of the absorption cross section in this way. In this work, calculating the absorption cross section, we use only a normalizable mode Eq.(20) within the southern diamond (SD). Hence we do not have enough information to determine the absorption cross section in the bulk spacetime approach. Fortunately, using the universality for s-wave cross section in the black hole background, we could determine the absorption cross section. This also happens in the study of the AdS-black holes [22].

Finally we wish to mention that the bulk absorption cross section can be also calculated from the two-point function of CFT defined on the boundary if one assumes the AdS/CFT correspondence to realize the holographic principle [23]. Hence we hope that our results for bulk dS space can be recovered from the CFT approach $[8,24,25]$ by making use of the dS/CFT correspondence to realize the assumed dS holography. Also the dS complementarity principle may help to remedy the undetermined normalization factor in Eq.(29) [26]. Consequently the exact form of the absorption cross section for the cosmological horizon will be found if one gets the CFT results.

\section{ACKNOWLEDGEMENT}

We thank Hyung Won Lee for helpful discussions. This work was supported in part by KOSEF, Project Nos. R01-2000-000-00021-0 and R02-2002-000-00028-0. 


\section{REFERENCES}

[1] S. Perlmutter et al.(Supernova Cosmology Project), Astrophys. J. 483, 565(1997)[astroph/9608192].

[2] R. R. Caldwell, R. Dave, and P. J. Steinhard, Phys. Rev. Lett. 80, 1582(1998)[astro$\mathrm{ph} / 9708069]$.

[3] P. M. Garnavich et al., Astrophys. J. 509, 74(1998)[astro-ph/9806396].

[4] E.Witten, "Quantum Gravity in de Sitter Space", hep-th/0106109.

[5] S. Hellerman, N. Kaloper, and L. Susskind, JHEP 0106, 003(2001)[hep-th/0104180].

[6] W. Fischler, A. Kashani-Poor, R. McNees, and S. Paban, JHEP 0107, 003 (2001)[hepth/0104181].

[7] R.Bousso, JHEP 0011, 038 (2000)[hep-th/0010252]; R. Bousso, JHEP 0104, 035 (2001)[hep-th/0012052]; S. Nojiri and D. Odintsov, Phys.Lett. B519, 145 (2001)[ hepth/0106191]; D. Klemm, "Some Aspects of the de Sitter/CFT correspondence", hepth/0106247 ; M. Spradlin, A. Strominger, and A. Volovich, "Les Houches Lectures on De Sitter Space", hep-th/0110007; S. Cacciatori and D. Klemm, "The Asymptotic Dynamics of de Sitter Gravity in three Dimensions", hep-th/0110031; A. C. Petkou and G. Siopsis, "dS/CFT correspondence on a brane", hep-th/0111085. V. Balasubramanian, J. de Boer, and D. Minic, "Mass, Entropy and Holography in Asymptotically de Sitter Spaces", hep-th/0110108; R. G. Cai, Y. S. Myung, and Y. Z. Zhang, "Check of the Mass Bound Conjecture in de Sitter Space", hep-th/0110234; Y. S. Myung, Mod. Phys. Lett.A16, 2353(2001)[hep-th/0110123]; R. G. Cai, "Cardy-Verlinde Formula and Asymptotically de Sitter Spaces", hep-th/0111093; A. M. Ghezelbach and R. B. Mann, JHEP 0201, 005 (2002)[hep-th/0111217]; M. Cvetic, S. Nojiri, and S.D. Odintsov, "Black Hole Thermodynamics and Negative Entropy in deSitter and Anti-deSitter Einstein-Gauss-Bonnet gravity", hep-th/0112045; Y. S. Myung, "Dynamic dS/CFT correspondence using the brane cosmology", hep-th/0112140; R. G. Cai, "Cardy-Verlinde Formula and Thermodynamics of Black Holes in de Sitter Spaces", hep-th/0112253.

[8] A. Strominger, JHEP 0110, 034 (2001)[hep-th/0106113]; A. J. Tolley and N. Turok, "Quantization of the massless minimally coupled scalar field and the dS/CFT correspondence", hep-th/0108119.

[9] C. Callan, S. Gubser, I. Klebanov, and A. Tseytlin, Nucl. Phys. B489, 65(1997)[hepth/9610172]; M. Karsnitz and I. Klebanov, Phys. Rev. D56, 2173(1997)[hepth/9703216]; B. Kol and A. Rajaraman, Phys. Rev. D56, 983(1997)[hep-th/9608126]; M. Cvetic and F. Larsen, Nucl. Phys. B506, 107(1997)[hep-th/9706071].

[10] A. Dhar, G. Mandal, and S. Wadia ,Phys. Lett. B388, 51(1996) [hep-th/9605234]; S. Das, G. Gibbons and S. Mathur, Phys. Rev. Lett. 78, 417(1977)[hep-th/9609052].

[11] M. I. Park, Phys.Lett. B440, 275(1998) [hep-th/9806119]; M. Banados, T. Brotz and M. Ortiz, Phys. Rev. D59, 046002(1999)[hep-th/9807216]; W. T. Kim, Phys. Rev. D59, 047503(1999)[hep-th/9810169]; F. Lin and Y. Wu, Phys. Lett. B453, 222 (1999)[hepth/9901147].

[12] W. T. Kim, J.J. Oh, and K. H. Yee, "Scattering amplitudes and thermal temperatures of the Schwarzschild-de Sitter black holes", hep-th/ 0201117.

[13] H. Suzuki, E. Takasugi, and H. Umetsu, Prog. Theor. Phys. 103, 103 (2000)[grqc/9911079]. 
[14] H. W. Lee and Y. S. Myung, Phys. Rev. D61, 024031(2000)[hep-th/9903054]; H. W. Lee, Y. S. Myung, and J. Y. Kim, Phys. Rev. D58, 104006(1998)[hep-th/9708099].

[15] R. Bousso, A. Maloney, and A. Strominger, "Conformal vacua and entropy in de Sitter space", hep-th/0112218.

[16] M. Abramowitz and I. Stegun, "Handbook of Mathematical Functions" (Dover Publicaton ,New York, 1970), p.564.

[17] H. Suzuki and E. Takasugi, Mod. Phys. Lett. A11, 431(1996)[gr-qc/9508068].

[18] S. Gubser and Hashimoto, Commun.Math. 203, 325(199)[hep-th/9805140].

[19] J. Madacena and A. Strominger, Phys. Rev. D56, 4975(19970[hep-th/9702015]; S. D. Mathur, Nucl. Phys. B514, 204(1998)[hep-th/9704156].

[20] H.-T. Sato and H. Suzuki, Mod. Phys. Lett. A9, 3673 (1994)[hep-th/9410092].

[21] N. D. Birrell and P. C. W. Davies, Quantum fields in curved space (Cambridge Univ. Press, New York, 1982) p. 260.

[22] D. B. Birmingham, I. Sachs and S. Sen, Phys. Lett. B413, 281(1997); H.W. Lee, N. J. Kim, and Y. S. Myung, Phys. Rev. D58, 084022 (1988)[hep-th/9803080]; H.W. Lee, N. J. Kim, and Y. S. Myung, Phys. Lett. B441, 83(1988)[hep-th/9803227]; H.W. Lee and Y. S. Myung, Phys. Rev. D58, 104013 (1988)[hep-th/9804095].

[23] E. Teo, Phys. Lett. B436, 269(1988)[hep-th/9805014]; Y. S. Myung and H. W. Lee, JHEP 9910, 009(1999)[hep-th/9904056].

[24] M. Spradlin and A. Volovich, "Vacuum states and the S-matrix in dS/CFT", hepth/0112223.

[25] E. Abdalla, B. Wang, A. Lima-Santos and W. G. Qiu, "Support of dS/CFT correspondence from perturbations of three dimensional spacetime", hep-th/0204030.

[26] U. H. Danielsson, D. Domert and M. Olsson, "Miracles and complementarity in de Sitter space", hep-th/0210198. 